\title{
DYNASTIC CHRONOLOGY
}

THE CHRONOLOGY IS SELECTIVE, BASED ON RULERS AND periods mentioned in the text. Dates mostly follow Ian Shaw, ed., The Oxford History of Ancient Egypt (Oxford, 2000), 480-482, and Hölbl 2001.

Early Dynastic Period (Dynasties 1-2)

c. $3000-2686$ B.C.

Old Kingdom (Dynasties 3-6)

c. $2686-2181$

Middle Kingdom (Dynasties 11-13)

c. $2055-1650$

New Kingdom (Dynasties 18-20)

c. $1550-1069$

Third Intermediate Period (Dynasties 21-25)

c. $1069-664$

Late Period (Dynasties 26-30)

c. $664-332$

Dynasty 29

399-380

Dynasty 30

$380-343$

Second Persian Period

$343-332$

Macedonian Period

Alexander the Great

332-306 B.C.

Philip Arrhidaios

$332-323$

$323-317$

Alexander IV

317-310/309

Ptolemaic Period

306-30 B.C.

Ptolemy I Soter

$306-283 / 282$

Ptolemy II Philadelphos

285/284-246

Arsinoe II

279/274-270

Ptolemy III Euergetes I

$246-222 / 221$ 
Berenice II

Ptolemy IV Philopator

Arsinoe III

Ptolemy V Epiphanes

Cleopatra I

Ptolemy VI Philometor

Cleopatra II

Ptolemy VIII Euergetes II

Cleopatra III

Ptolemy IX Soter II

Ptolemy X Alexander I

Ptolemy XI Alexander II

Ptolemy XII Neos Dionysos

Cleopatra VII Philopator

Ptolemy XIII

Ptolemy XIV

Ptolemy XV Caesarion

Roman Period

Octavian-Augustus

Caracalla

$$
\begin{array}{r}
246-221 \\
221-204 \\
221-204 \\
204-180 \\
194 / 193-176 \\
180-145 \\
175-116 \\
170-163,145-116 \\
141 / 140-101 \\
116-107,88-80 \\
107-88 \\
80 \\
80-58,55-51 \\
51-30 \\
51-47 \\
47-44 \\
44-30
\end{array}
$$

3 B.C.-A.D. 395

3 O B.C.-A.D. 14

A.D. $198-217$ 
PORTRAITS OF THE PTOLEMIES 
THIS PAGE INTENTIONALLY LEFT BLANK 